\title{
11
}

\section{Conservation Laws for Dynamic Routing in Multi-path Networks}

\author{
Sandeep Sharma, \\ Alcatel Network Systems, \\ 2912 Wake Forest Rd., Raleigh, NC 27609, USA. \\ e-mail: sharma@aur.alcatel.com. \\ and \\ Ioannis Viniotis, \\ Electrical and Computer Engineering Department, \\ North Carolina State University, Raleigh, NC 27606, USA. \\ e-mail: candice@ececho.ncsu.edu.
}

\begin{abstract}
In this paper, we discuss the feasibility and synthesis aspects of routing algorithms, which have to meet a specified performance criterion. The queuing model for the routing algorithms involves a central router, multiple output queues, each with its own server, deterministic service times, and general, independent, arrival patterns. The performance criterion is the loss probability at each queue, which is specified apriori, and which the router tries to achieve. We analyze this problem using Conservation Laws, and propose a dynamic routing algorithm which tries to achieve the specified criterion. Under certain assumptions, the same theory can be applied to the general class of load balancing problems.
\end{abstract}

\section{Introduction}

The queuing model studied in this paper is representative of a collection of computer nodes providing distributed access to a communication network. For such a communication network, where multiple paths might exist between a given source and destination pair, a routing decision has to be made at a node, regarding which path the next packet gets sent on from the source. It is important that such a decision provides the source an acceptable quality of service (in terms of cell loss) along each of these paths.

Typically, the algorithms these routers use try to minimize average response times, blocking probabilities, equalize server idle time, etc. Also, these algorithms can be classified as either static or dynamic, depending on whether the routing decision depend on the instantaneous state (state is defined to be the vector of the number in each queue in the system) of the system or not. Previous research shows that dynamic algorithms offer 


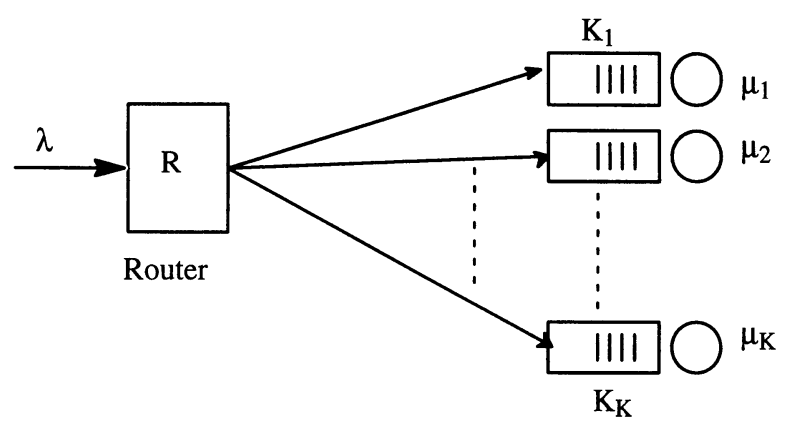

Figure 1: The queuing model.

better performance than static ones [7]. The efficiency of these heuristics, which need to maintain state information, is afforded at an increased expense of trying to gather this state information [3].

In this paper, we propose routing algorithms using Conservation Laws [2]- [5]. These laws embody the principle that improvement of performance at one queue, is generally afforded by a corresponding degradation at another [2]. Conservation laws have been used to propose algorithms for achieving loss and delay objectives for single-queue systems [2][5]. But the idea of using the same for the design of routers is new. We use this idea to propose a Conservation Region, which encloses the set of all achievable loss probabilities for the queuing model explained in Section 2. Based on the conservation region, we then propose static and dynamic routing algorithms, which try to achieve a specified loss criterion.

Although we focus on loss probabilities, maintaining the state information effectively balances the load and minimizes response times also. Hence, in addition to achieving a specified loss criterion, the router can also efficiently distribute the load presented to it. Therefore, under certain assumptions, the same theory can be applied to the general class of load balancing problems.

The rest of the paper is laid out as follows: The following section provides a brief description of the problem, and the associated queuing model. Section 3 presents the theory associated with the design of an optimal router. Section 4 provides conservation laws for loss probabilities. In Sections 5 and 6 we present static and dynamic algorithms, which try to achieve a specified loss performance. Finally, Section 7 concludes this paper.

\section{The Queuing Model}

The queuing model for the problem under study is shown in Figure 1. This model is representative of $K$ communication links, each with a finite sized buffer of size $K_{i}$ in front of it. A router, $R$, routes incoming packets to one of the $K$ communication links. Arrivals to the router are assumed to have a general, independent inter-arrival time distribution, 
and have a mean rate $\lambda$ packets/sec. Server $i$ is assumed to have a deterministic service rate of $\mu_{i}$ bits/sec. The service time for each packet is assumed to be deterministic. (This holds true for the fixed size Asynchronous Transfer Mode (ATM) cells.) The problem being studied can be applied to an ATM network, where multiple Virtual Circuits/Paths (VC/Ps) exist between a given source-destination pair. Applications which need a stricter loss guarantee, not provided by any one of the VC/Ps, may choose to distribute their load amongst several such $\mathrm{VC} / \mathrm{Ps}^{*}$.

Our solution assumes that the router has knowledge of the state of the different queues, i.e., it knows the instantaneous number in each of the queues. As mentioned before, several papers in the literature comment on the overhead of tracking this instantaneous number [1]. One stated way of tracking this number is by having the nodes send back status messages [3], informing the router of the instantaneous number in the queue. In addition to the overhead involved with this method, such information is quickly outdated. Other papers resort to prediction mechanisms [3], which predict the instantaneous number at routing decision instants. Due to the deterministic service times of the packets, and assuming that the router knows the processing rate of each link, the router can theoretically track the instantaneous number in each of the queues, based on the number of packets that it routes to them. This potentially does away with the status messages that are needed to inform the router of the instantaneous number in the queue. Other constraints that are placed on the router are that an arriving packet is not discarded by the router (i.e., it has to route it to one of the queues), and that the router does not influence the arrival and service times of packets.

\section{Design of an Optimal Router}

In this section, we present the design of an optimal router, which minimizes the total loss in the system. To aid us in our discussion, we introduce the following proposition:

Proposition 1: An optimal router, $R_{o}$, which tries to minimize the loss probability at the queues ahead of it, discards packets only if all queues are full.

We prove this proposition using sample path arguments [9]. In Appendix I, we show that if there exist queues which have space in them, a decision to drop the packet can, at most, achieve a loss probability which is greater than or equal to the loss probability achieved by accepting the packet.

Assuming $R_{o}$ follows Proposition 1, we further constrain the behaviour of $R_{o}$ in its choice of queue to route the packet to, in case there exist multiple queues which can accept an arriving packet. The main objective for doing so is to arrive at a lower bound for the loss probabilities that can be achieved at any of the queues. We define $N_{i}\left(t, R_{o}\right)$ to be the number of packets in queue $i$ at time $t$, such that $N_{i}\left(t, R_{o}\right) \leq K_{i}$, the buffer size of queue $i$. Also, let $R_{i}\left(t, R_{o}\right)$ be the residual service time of the packet in service at queue $i$. Defining the work in queue $i, W_{i}\left(t, R_{o}\right)$, as the sum of the remaining service times of

\footnotetext{
*Note that sequencing the packets distributed across the VC/Ps is an application level function (which is trying to space out the packets over multiple $\mathrm{VC} / \mathrm{Ps}$ ).
} 
the packets in the queue and in service, and assuming that the size of each packet is $T$ bits, $W_{i}\left(t, R_{o}\right)$ can be written as:

$$
W_{i}\left(t, R_{o}\right)=\frac{T \cdot N_{i}\left(t, R_{o}\right)}{\mu_{i}}+R_{i}\left(t, R_{o}\right)
$$

For a 'tagged' packet arriving at time $t$, the response time, $T_{i}\left(t, R_{o}\right)$, that this packet sees at queue $i$ is:

$$
T_{i}\left(t, R_{o}\right)=W_{i}\left(t, R_{o}\right)+\frac{T}{\mu_{i}}
$$

We now introduce the following theorem, which is the main result of this section:

Theorem 1: For minimizing loss probabilities, an optimal router chooses a queue $i$, based on the following criterion:

$$
\min _{\forall i} T_{i}\left(t, R_{o}\right) \quad i_{s t}, N_{i}\left(t, R_{o}\right) \neq K_{i}
$$

Proof: If $i$ is selected from the above criterion, the response time of the tagged packet will be the least (since the packet is being sent to a queue where it has the least instantaneous response time). If the above specified criterion is followed for all arriving packets, the average response time of these packets will also be the least possible under any routing algorithm $R$. Using Little's Law ${ }^{\dagger}$, it can be concluded that the average number in the system of queues will also be the least. This implies that the available buffer space is maximized under such a policy. Since packets are lost only when all the buffers in the system are full, we have proved that a policy following the rule specified by Equation (3) minimizes the number of packets lost in the system of queues.

Thus, for minimizing the number of packets lost, $R_{o}$ is constrained to follow Equation (3). By following a policy that minimizes the number of packets lost, the router also has a finer control over the loss probabilities that can be achieved at any of the queues. This shall be further explained in the next section.

\section{Conservation Region for Achievable Loss Probabilities}

In this section, we present the region of achievable loss probabilities at different queues. We present lower and upper bounds on achievable loss probabilities, and then combine the two to arrive at a Conservation Region, which encompasses the set of all achievable points.

\footnotetext{
'Little's Law states that for an average arrival rate and waiting time of $\lambda$ and $T$ respectively, the average number, $N$, in the ergodic system, can be written as $N=\lambda \cdot T$. For further details, refer [11].
} 


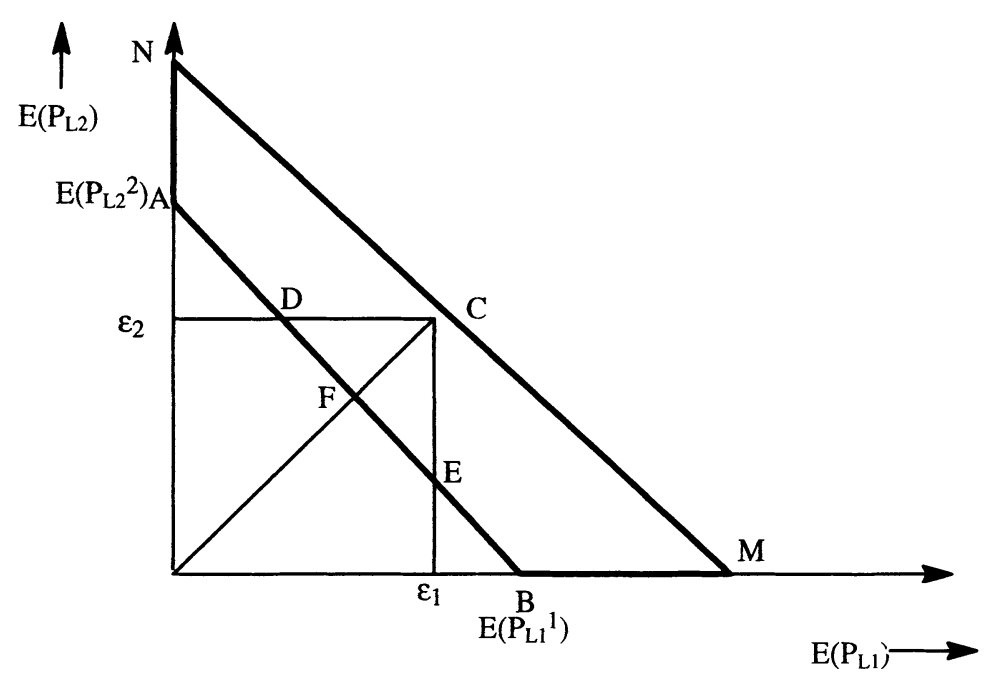

Figure 2: Conservation Region for two queues.

For our discussion, we define the start of a busy period as the time instant when the first packet enters an idle system of queues, and define the time instant when a packet leaves behind an idle system of queue to be the end of a busy period.

\subsection{A lower bound on achievable loss probabilities}

As proved earlier, the optimal router $R_{o}$ minimizes the number of packets lost in the system of queues. Thus, for achieving lower bounds for loss probabilities, we assume $R_{o}$ to be the router. In Appendix II, we derive the following relationship, which gives us $E\left(P_{L i}\right)$, the average loss probability at queue $i$ :

$$
E\left(P_{L i}\right)=\alpha_{i} E\left(P_{L i}^{i}\right)
$$

where $\sum_{i=1}^{K} \alpha_{i}=1, \alpha_{i} \geq 0$, and $E\left(P_{L i}^{i}\right)$ is the average loss probability for queue $i$ when packets are lost only at queue $i$. Since packets are lost only when all queues are full, $E\left(P_{L i}^{i}\right)$ denotes the maximum loss probability at queue $i$, assuming that all these packets are lost only at queue $i$.

We now apply this result to the case where $K=2$. Refer to Figure 2 where loss probabilities for the two queues are plotted. Assuming that the optimal routing algorithm is used, the average loss probability at each of the queues, as given by Equation (4), can be written as: 


$$
\begin{array}{r}
E\left(P_{L 1}\right)=\alpha_{1} E\left(P_{L 1}^{1}\right) \\
E\left(P_{L 2}\right)=\left(1-\alpha_{1}\right) E\left(P_{L 2}^{2}\right) .
\end{array}
$$

Equations (4)-(6) constrain the minimum achievable loss probabilities for both the queues to lie on line A-B. A value of $\alpha_{1}=0$ achieves point $\mathrm{A}$. This effectively means that the minimum number of packets that would be lost under $R_{o}$, would all be lost at queue 2. A value of $\alpha_{1}=1$ achieves point $\mathrm{B}$, which means that the minimum number of packets that would be lost under $R_{o}$, would all be lost at queue 1 . Any other value of $\alpha_{1}$ gives a point which lies on line A-B. (This would mean that the packets which are lost are 'distributed' amongst the two queues.) This behaviour forms the basis of the Conservation Law, which states that to decrease the loss probability at one queue, you see an increase at the other. Note that under $R_{o}$, line A-B is the lower bound on achievable loss probabilities at either of the two queues.

\subsection{An upper bound on achievable loss probabilities}

Consider the following routing policy: With busy periods as defined before, if the packet which starts a busy period is sent to queue $i$, all subsequent packets in that busy period are also sent to queue $i$. The choice of $i$ is made at the beginning of busy periods.

The length of a busy period depends upon the rate of server $i$, as well as the available buffer at queue $i$. Under such a policy, the loss at queue $i$ is maximum in that busy period. No other policy can achieve a higher loss at queue $i$, since all arriving packets are sent to only one queue (hence making this policy different from $R_{o}$ ). It is easy to note that the average loss probability at queue $i$ can be written as:

$$
E\left(P_{L i}\right)=\alpha_{i}^{\prime} E\left(P_{L i}^{i}\right)^{\prime}
$$

where $\alpha_{i}^{\prime}$ is the fraction of busy periods when all arriving packets are routed to queue $i$, and $E\left(P_{L i}^{i}\right)^{\prime}$ is the average loss probability at queue $i$, during these busy periods.

Applying this result to two queues, we get line $\mathrm{M}-\mathrm{N}$ in Figure 2. Point $\mathrm{M}(\mathrm{N})$ corresponds to routing all the arriving traffic to queue $1(2)$. Since no other point above the line segment $\mathrm{M}-\mathrm{N}$ is achievable, this line provides an upper bound on the achievable loss probabilities.

\subsection{Conservation Region for two queues}

In Figure 2, Region A-B-M-N provides us with a Conservation Region. Since any point in this region can be represented by a linear combination of points $A, B, C$, and D, all points in this region are achievable. However, since A-B is the lower bound, if a goal which resides above line A-B is specified (anywhere in region A-B-M-N), a corresponding point on Line A-B would not only satisfy the specified goal, but even do better. Hence, for further discussion, we restrict our policies to achieve points on line $A-B$, since there exists a point on line A-B, which can satisfy any point in Region A-B-M-N. 
We use this result in the next two sections to propose static and dynamic algorithms which try to achieve a specified loss probability at different queues.

\section{Static Algorithms}

Section 4 gave the framework for achievability of loss probabilities. In this section, we look at the synthesis aspect. We consider a static algorithm $\left(R_{o}\right.$ in nature), which decides which queue will suffer a loss of packet, if all queues are full. This decision process is driven by having the average loss at queue $i, E\left(P_{L i}\right)$, be less than or equal to a specified performance criterion, $\epsilon_{i}$. This is known apriori, and can be stated as:

$$
E\left(P_{L i}\right) \leq \epsilon_{i}
$$

For two queues, this translates into point $\mathrm{C}$ in Figure 2. Let point $\mathrm{A}$ be achieved by a router $r_{1}$ which incurs losses only at queue 1 (similarly $r_{2}$ for point B). Each point on line A-B can be achieved by using a mixing policy $\left(\alpha, r_{1}, r_{2}\right)$ [5], which operates as follows:

Algorithm S: At the beginning of each busy period, the router follows rule $r_{1}$ with probability $\alpha$, and rule $r_{2}$ with probability $(1-\alpha)$. The rule which is chosen is followed throughout a busy period.

The achievable losses, as derived in Section 4, are:

$$
\begin{array}{r}
E\left(P_{L 1}\right)=\alpha E\left(P_{L 1}^{1}\right) \\
E\left(P_{L 2}\right)=(1-\alpha) E\left(P_{L 2}^{2}\right) .
\end{array}
$$

By a suitable choice of $\alpha$, all points on line A-B can be achieved (ref: Section 4). Hence, given a goal $\mathrm{C}$, a suitable choice of $\alpha$ can give us any one of the infinite points on line segment D-E, which satisfy our criterion. The appropriate value of $\alpha$ can be computed by the initial basis Linear Programming method [2]. As pointed out in [5], this problem is easy to solve for a few queues, but computationally expensive for more. Another drawback of this method is that $\alpha$ is static. Therefore, the router cannot respond to overload periods. Moreover, as the router makes decisions only at the beginning of busy periods, which may not be very frequent if the length of the busy periods is long, there can potentially be very high variances between the actual packet loss probabilities ( 0 in one case, and $E\left(P_{L i}^{i}\right)$ in the other). Besides this, the static method also warrants knowing the values of the points $A$ and $B$. This can potentially be a problem, since these points have to be either computed via simulation, or determined analytically. In the next section, we propose a dynamic algorithm which essentially does away with the above mentioned drawbacks. 


\section{Dynamic Algorithms}

In this section, we propose a dynamic algorithm for achieving the performance criterion given by Equation (7). In doing so, we follow the approach in [5]. This algorithm continuously monitors the system performance and bases its decisions on the state of the system. Since Equation (7) may not have a unique solution (all points on line segment D-E satisfy the specified criterion), we introduce the following criterion [5]:

$$
\min \max _{i} \frac{E\left(P_{L i}\right)}{\epsilon_{i}} \quad \forall i
$$

The criterion stated in Equation (9) achieves a unique point on line segment D-E in Figure 2. Criterion 9 tries to equalize the $\frac{E\left(P_{L i}\right)}{\epsilon_{i}}$ s. The idea behind using the above criterion is to be able to dynamically decide which queue should be chosen to lose packets so that the specified loss probabilities can be achieved. That is, if the loss probability at a queue exceeds its specified goal, but that at another queue does not, by following the above criterion, packets will be lost at the latter queue. The point that is achieved is point $\mathrm{F}$, which is optimal for the above criterion [4].

To implement Criterion 9 , we define $G_{i}(t)$, a performance index for each queue, which takes into account the goal to be achieved, and the current performance that is being achieved. With $N_{L i}(t)$ and $N_{T i}(t)$ denoting the total number of packets lost/routed at/to queue $i$ in time $[0, t], G_{i}(t)$ can be written as:

$$
G_{i}(t)=\frac{P_{L i}(t)}{\epsilon_{i}}
$$

where $P_{L i}(t)=\frac{N_{L i}(t)}{N_{T i}(t)}$. With $G_{i}(t)$ s defined by Equation (10), we propose the following algorithm:

Algorithm D: For the duration of a busy period, packets shall be lost at the queue which has the smallest value of $G_{i}(t)$. (As pointed out in Appendix II, this decision can be made at each discard instant also.)

We now describe the dynamic behaviour of this algorithm. Consider Figure 3, which plots $G_{i}(t)$ instead of $E\left(P_{L i}\right)$, for a given $\epsilon_{i}$. Here, line A-B is a mapping of line A-B in the $P_{i}$ plane shown in Figure 2. All achievable points, at steady state, should lie on line A-B. Also, since the proposed algorithm equalizes the $G_{i}(t) \mathrm{s}$, it forces the achieved point to lie on line O-C, which is the line of equality for the $G_{i}(t)$ s. In Figure 3, all points in Region 1 have $G_{1}$ greater than $G_{2}$ (similarly, $G_{2}>G_{1}$ in Region 2). Assume $X$ is an initial starting point for the router. Since, $G_{1}$ is greater than $G_{2}$, at a decision instant, the router will choose queue 2 for discarding packets. This would cause the current achievable point to 'drift' towards Region 2. A similar kind of phenomenon in Region 2 would cause the achievable point to drift back to Region 1 . Since the achievable point needs to lie on both $\mathrm{O}-\mathrm{C}$ and $\mathrm{A}-\mathrm{B}$, the algorithm would eventually cause the achievable point to drift towards the point of intersection of both the line segments. This kind of a drift behaviour has 


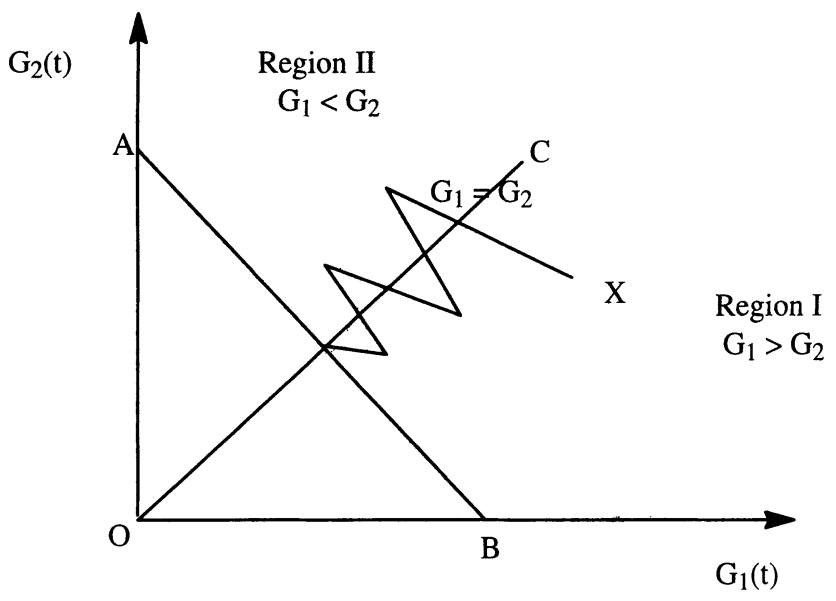

Figure 3: Conservation Region for two queues in the $G_{i}$ plane.

been analyzed in [4], and it has been shown that the steady state point that is achieved is indeed the point of intersection of line A-B, and O-C.

The algorithm proposed above is min-max fair. If a specified goal in unachievable, i.e., the point $\mathrm{C}$ lies below line $\mathrm{A}-\mathrm{B}$, it achieves a point on line $\mathrm{A}-\mathrm{B}$ which is best for both classes (the point of intersection of O-C with A-B).

Since the $G_{i}(t)$ s are dynamically computed and updated, this algorithm is more responsive in overload periods than the static algorithms. Also, this algorithm does not need the values of the $\mathrm{A}, \mathrm{B}, \mathrm{M}$, or $\mathrm{N}$ points. This is a very big advantage, since these points are not easily computable for most arrival patterns.

\section{Conclusions}

We have proposed the use of Conservation Laws for achieving a specified set of loss probabilities for different queues. We present a dynamic algorithm, min-max-fair in behaviour, which helps us achieve this objective. It does not suffer from the drawbacks that the static algorithms suffer from. Also, due to the deterministic service time assumption, the router keeps track of the state of the system, and routes to the queue where the response time is the least. This is an optimal way for load balancing, and minimizes the total number of packets that are lost in the system of queues in any given time interval. 


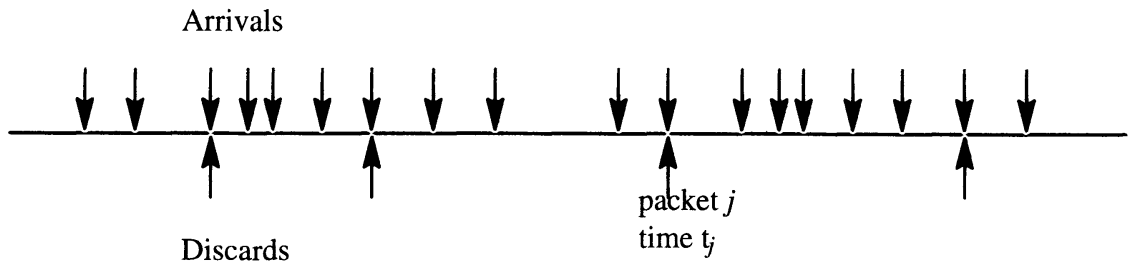

Figure 4: Time instants for discarding packets.

\section{Appendix I}

In this appendix, we present the proof for Proposition 1, which states that an optimal router, $R_{o}$, which tries to minimize the loss probability at the queues ahead of it, discards packets only if all queues are full.

Proof: In order to facilitate our discussion, we introduce the idea of an arbitrary router, and focus on time instants that this router discards packets. This concept is reflected in Figure 4, which also embodies a sample path of arriving packets. Here, the arbitrary router discards packet $j$ at time instant $t_{j}$. These discard instants, for the arbitrary router, are restricted to be one of the following:

1. All queues are full, and the packet is lost at one of the queues.

2. At least one of the queues can accept the packet, and the packet is lost at a queue which is full.

The above conditions impose a restriction on the arbitrary router: it has to send an arriving packet to one of the queues.

We now proceed to prove that an optimal router discards packets only if all queues are full. To aid in our explanation, let:

$R_{a}\left(R_{o}\right)$ be the arbitrary (optimal) router

$N_{L i}\left(t, R_{a}\right)\left(N_{L i}\left(t, R_{o}\right)\right)$ be the number of packets lost at queue $i$ under routing policy $R_{a}$ $\left(R_{o}\right)$ in time $[0, t]$

$N_{i}\left(t, R_{a}\right)\left(N_{i}\left(t, R_{o}\right)\right)$ be the number of packets routed to queue $i$ under routing policy $R_{a}$ $\left(R_{o}\right)$ in time $[0, t]$ 
$N_{i}^{S}(t)$ be the number of packet saved by $R_{o}$ over $R_{a}$, at queue $i$ in time $[0, t]$. These packets would have normally been lost under $R_{a}$, but instead were saved by routing them to a non-full queue.

$N_{i}^{F}(t)$ be the number of packets routed to queue $i$ instead of being discarded at a queue which was full (assuming queue $i$ had space in it). Whether these packets will be saved by $R_{o}$ over $R_{a}$ is not known at time $t$.

$P_{L i}\left(t, R_{a}\right)\left(P_{L i}\left(t, R_{o}\right)\right)$ be the loss probability at queue $i$ under routing policy $R_{a}\left(R_{o}\right)$ in time $[0, t]$

For the given sample path, Figure 4 also shows the time instances when $R_{a}$ discards packets. In order to compare the performance of $R_{o}$ with $R_{a}, R_{o}$ is defined to follow routing decisions made by $R_{a}$ at all arrival instants, except at time instances where $R_{a}$ discards packets. If all the queues are full at one of these time instances, $R_{o}$ shall follow $R_{a}$ 's decision. However, if there exists a queue $i$ which can accept the discarded packet (say packet $A$ ), $R_{o}$ shall route packet $A$ to this queue. One key observation: by accepting this packet which $R_{a}$ would have discarded, and by continuing to follow $R_{a}$ 's future routing decisions, it may happen that $R_{o}$ has to drop a packet (in the future), that $R_{a}$ accepts. If such a situation arises, and there exists no queue which can accept this new arrival, this packet shall be lost at the queue where $R_{a}$ lost packet $A$.

The variables $N_{L i}\left(t, R_{o}\right), N_{L i}\left(t, R_{a}\right), N_{i}^{S}(t)$, and $N_{i}^{F}(t)$, shall be used by $R_{o}$ for keeping track of the extra packets that it accepts. These variables shall change as follows: At a discard time instance for $R_{a}$, if all queues are full, and if $R_{a}$ routes the lost packet to queue $i, R_{o}$ shall also route the packet to queue $i$. Thus:

$$
\begin{gathered}
N_{L i}\left(t, R_{a}\right)=N_{L i}\left(t, R_{a}\right)+1 \\
N_{L i}\left(t, R_{o}\right)=N_{L i}\left(t, R_{o}\right)+1
\end{gathered}
$$

If at a discard time instance for $R_{a}$, there is at least one queue which is non-full, and if $R_{a}$ routes it to queue $i$ (since it is a discard instant, queue $i$ is full), $R_{o}$ shall route it to queue $j$ which is non-full. The variables shall be updated as follows:

$$
\begin{array}{r}
N_{L i}\left(t, R_{a}\right)=N_{L i}\left(t, R_{a}\right)+1 \\
N_{j}^{F}(t)=N_{j}^{F}(t)+1 .
\end{array}
$$

The reason for incrementing $N_{j}^{F}(t)$ is that this packet is an extra arrival to queue $j$. This extra arrival might cause a future packet to get dropped from queue $j$. Hence, at this time, since the future impact of accepting this packet cannot be predicted for sure, the variable reflecting, 'Will know in future' gets incremented.

If at all $R_{o}$ encounters an arrival which $R_{a}$ routes to a non-full queue $i$, but $R_{o}$ finds full because of accepting previous packets $\left(N_{i}^{F}(t)\right.$ should be greater than zero), it does the following: 
1. If there exists a non-full queue $j, R_{o}$ routes this packet to queue $j$, and:

$$
\begin{gathered}
N_{i}^{F}(t)=N_{i}^{F}(t)-1 \\
N_{j}^{F}(t)=N_{j}^{F}(t)+1
\end{gathered}
$$

By decrementing $N_{i}^{F}(t)$, we effectively nullify the acceptance of a prior extra packet.

2. If all queues are full, find a queue, $j$, for which $N_{L j}\left(t, R_{a}\right)>N_{L j}\left(t, R_{o}\right)$, and route this packet to that queue. (Again, this can only happen if $N_{i}^{F}(t)>0$.) Also, adjust the variables such that:

$$
\begin{array}{r}
N_{i}^{F}(t)=N_{i}^{F}(t)-1 \\
N_{L j}\left(t, R_{o}\right)=N_{L j}\left(t, R_{o}\right)+1 .
\end{array}
$$

The reason for decrementing $N_{i}^{F}(t)$ is the same as before.

Now, if at all a queue $i$ sees extra arrivals (like when $R_{o}$ routes a packet to a non-full queue rather than discarding it), these arrivals cannot be 'saved' unless the number in that queue comes down to zero. The reason for this is that after accepting an extra packet, one future packet can always attribute its discarding to the acceptance of this extra packet. This, however, is not the case if the number in the queue comes down to zero, since the history of extra packets accepted is only maintained while the number in the queue is greater than zero. Hence, as soon as the number in the queue at any queue $i$ falls to zero, if $N_{i}^{F}(t)$ is greater than zero, $N_{i}^{F}(t)$ number of packets are truly saved by $R_{o}$ over $R_{a}$. Hence, at that time instant:

$$
\begin{array}{r}
N_{i}^{S}(t)=N_{i}^{S}(t)+N_{i}^{F}(t) \\
N_{i}^{F}(t)=0 .
\end{array}
$$

The resetting of $N_{i}^{F}(t)$ to zero effectively means the discarding of previous history.

We can write down the loss probabilities at each queue under both the routers as follows: Under $R_{a}$, the loss probability at queue $i$ is:

$$
P_{L i}\left(t, R_{a}\right)=\frac{N_{L i}\left(t, R_{a}\right)}{N_{i}\left(t, R_{a}\right)}
$$

Under $R_{o}$, the loss probability at queue $i$ is:

$$
P_{L i}\left(t, R_{o}\right)=\frac{N_{L i}\left(t, R_{o}\right)}{N_{i}\left(t, R_{a}\right)-N_{L i}\left(t, R_{a}\right)+N_{L i}\left(t, R_{o}\right)+N_{i}^{S}(t)+N_{i}^{F}(t)} .
$$


To compute the number of arrivals to the $i$ th queue, we use the total number of arrivals under $R_{a}$, and then subtract the number lost at queue $i$ under $R_{a}$, add the number lost under $R_{o}$, and finally, add the number saved, and the 'future' number under $R_{o}$.

The following invariance holds, effectively accounting for all the lost packets under $R_{a}$ :

$$
\sum_{i=0}^{i=K} N_{L i}\left(t, R_{a}\right)=\sum_{i=0}^{i=K}\left(N_{L i}\left(t, R_{o}\right)+N_{i}^{S}(t)+N_{i}^{F}(t)\right)
$$

The difference between $P_{L i}\left(t, R_{a}\right)$ and $P_{L i}\left(t, R_{o}\right)$ is:

$$
\delta P_{L i}(t)=\frac{\left[N_{L i}\left(t, R_{a}\right)-N_{L i}\left(t, R_{o}\right)\right]\left[N_{i}\left(t, R_{a}\right)-N_{L i}\left(t, R_{a}\right)\right]+N_{L i}\left(t, R_{a}\right)\left[N_{i}^{S}(t)+N_{i}^{F}(t)\right]}{N_{i}\left(t, R_{a}\right)\left[N_{i}\left(t, R_{a}\right)-N_{L i}\left(t, R_{a}\right)+N_{L i}\left(t, R_{o}\right)+N_{i}^{S}(t)+N_{i}^{F}(t)\right]} .
$$

Equation (14) is the delta-function of the loss probabilities under $R_{a}$ and $R_{o}$. Since $N_{L i}\left(t, R_{o}\right) \leq N_{L i}\left(t, R_{a}\right), N_{i}^{S}(t) \geq 0$, and $N_{i}^{F}(t) \geq 0$, the minimum value for Equation (14) is 0 . If the value of this function is uniformly zero, then $R_{a}=R_{o}$. This function can never take on a negative value. If $N_{i}^{S}(t)>0$, or $N_{i}^{F}(t)>0$, from Equation (13), $N_{L i}\left(t, R_{o}\right)<N_{L i}\left(t, R_{a}\right)$, and Equation (14) takes on a positive value. Hence, if the arbitrary router decides to discard a packet with non-full queues, the above function shall take on a positive value (if it doesn't, $R_{a}=R_{o}$ ). Future arrival patterns might force this function to go back to zero. The finite interval in which the above function takes on a positive value, contributes to making the performance of $R_{o}$ better than $R_{a}$, even though it may be for a small period of time.

This proves that a router $R_{o}$, by discarding packets only when all queues are full, performs better than any arbitrary router that doesn't.

\section{Appendix II}

In this appendix, we derive Equation (4). Let:

$N_{L j}^{i}$ be the number of packets lost at queue $i$ during the $j$ th busy period, given that the router decides that all losses will be incurred at queue $i$ during the $j$ th busy period.

$N_{j}^{i}$ be the number of packets routed to queue $i$ during the $j$ th busy period. (It is important to note that under $R_{o}, N_{L j}^{i}$ will be the same for any $i$. The same, however, does not hold for $N_{j}^{i}$.)

$t_{j}$ be the time instant signifying the end of the $j$ th busy period.

$B_{j}^{i}$ be the number of busy periods, in time $\left[0, t_{j}\right]$, in which losses are incurred at queue $i$ only.

$B_{j}^{T}$ be the total number of busy periods in time $\left[0, t_{j}\right]$. 
Since $R_{o}$, as presented in the previous section, is an optimal router for minimizing loss probabilities, we assume that the router has to follow $R_{o}$ for achieving a lower bound on loss probabilities.

We have the following equation for $E\left(P_{L i}\right)$, the average loss probability at queue $i$ till the end of the $B_{j}^{T}$ th busy period:

$$
E\left(P_{L i}\right)=\frac{\sum_{j=1}^{B_{j}^{T}} \frac{N_{L j}^{i}}{N_{j}^{i}}}{B_{j}^{T}}
$$

Or:

$$
E\left(P_{L i}\right)=\frac{\sum_{j=1}^{B_{j}^{i}} \frac{N_{L_{j}}^{i}}{N_{j}^{i}}+\sum_{j=1}^{B_{j}^{T}-B_{j}^{i}} \frac{N_{L_{j}}^{i}}{N_{j}^{i}}}{B_{j}^{T}} .
$$

During the $\left(B_{j}^{T}-B_{j}^{i}\right)$ busy periods, by definition there are no losses at queue $i$. Hence, the above equation can be rewritten as:

$$
E\left(P_{L i}\right)=\frac{\sum_{j=1}^{B_{j}^{i}} \frac{N_{L j}^{i}}{N_{j}^{i}}}{B_{j}^{T}} .
$$

Or:

$$
E\left(P_{L i}\right)=\frac{B_{j}^{i}}{B_{j}^{T}} \frac{\sum_{j=1}^{B_{j}^{i}} \frac{N_{L_{j}}^{i}}{N_{j}^{i}}}{B_{j}^{i}} .
$$

In Equation (18), $N_{L j}^{i}, N_{j}^{i}$ are iid random variables whose values in a busy period are independent of past busy periods for queue $i$. As $t \rightarrow \infty, B_{j}^{i} \rightarrow \infty$, and assuming that the system is ergodic and steady state averages exist, the Strong Law of Large Numbers [11] can be applied to Equation (18), giving us Equation (4), where $\alpha_{i}=\frac{B_{j}^{i}}{B_{j}^{T}}$, is the fraction of busy periods where losses occur at queue $i$, and $E\left(P_{L i}^{i}\right)$ is the average loss probability for queue $i$ during a busy period where packets are lost only at queue $i$.

Since the routing algorithm is optimal, Equation (4) provides a strict lower bound on the achievable loss probabilities at any queue $i$.

In the above discussion, we have assumed that all packets that are lost in a busy period, are all lost at only one of the $K$ queues. However, it is possible to distribute all packets lost in a busy period amongst all queues and still end up with a relation which is similar to Equation (6). To prove this statement, let $N_{L j}^{i}, N_{j}^{i}$ be defined as the number of packets lost/arriving at queue $i$ in the $j$ th busy period. The loss probabilities can now be written as: 


$$
E\left(P_{L i}\right)=\frac{\sum_{j=1}^{B_{T}} N_{L j}^{i}}{\sum_{j=1}^{B_{T}} N_{j}^{i}}
$$

Or:

$$
E\left(P_{L i}\right)=\frac{\sum_{j=1}^{B_{T}} N_{L j}^{i}}{\sum_{i=1}^{K} \sum_{j=1}^{B_{T}} N_{L j}^{i}} \cdot \frac{\sum_{i=1}^{K} \sum_{j=1}^{B_{T}} N_{L j}^{i}}{\sum_{j=1}^{B_{T}} N_{j}^{i}}
$$

Or:

$$
E\left(P_{L i}\right)=\alpha_{i}^{\prime} \cdot E\left(P_{L i}^{i}\right)
$$

where $\alpha_{i}^{\prime}$ is the fraction of the total packets that are lost at queue $i$ (and is different than $\alpha_{i}$ in Equation (4)), and $E\left(P_{L i}^{i}\right)$ is as defined earlier. Note that the achievable points are still constrained to lie on line A-B in Figure 2. Hence, rather than discard packets at queue $i$ for $\alpha_{i}$ busy periods, $\alpha_{i}^{\prime}$ of all lost packets can be discarded at queue $i$. The decision instants, however, are more frequent in the case where $\alpha_{i}^{\prime}$ packets are lost at queue $i$.

\section{References}

[1] Eager, D., Lazowska, E., Zahorjan, J. "A Dynamic Load Sharing in Homogeneous Distributed Systems", IEEE Trans. Software Engineering, Vol. 15, No. 12, May 1986.

[2] Gelenbe, E., Mitrani, I. "Analysis and Synthesis of Computer Systems", New York: Academic Press 1980.

[3] Goswami, K. K., Devarakonda, M., Iyer, R. K. "Prediction-Based Dynamic LoadSharing Heuristics", IEEE Transactions on Parallel and Distributed Systems, Vol. 4, No. 6, June 1993.

[4] Jeon, Y., Viniotis, I., Onuvural, R. "Conservation Laws and their Applications: Traffic Control in High Speed Networks", TR 29.1510, IBM Internal Report.

[5] Jeon, Y. "Conservation Laws and Multiplexing Schemes for Delay and Loss Requirements in Broadband Networks", PhD Thesis, Dept. of ECE, North Carolina State University, Raleigh, N.C., 1992.

[6] Lin, H., Raghavendra, C. S. "A Dynamic Load-Balancing Policy with a Central Job Dispatcher (LBC)", IEEE Transactions on Software Engineering, Vol. 18, No. 2, Feb. 1992.

[7] Liu, R., Wang, S. "Performance Modeling and Analysis of Load Balancing Policies with Priority Queuing", Journal of Systems Software, Vol. 20, 1993. 
[8] Silva, E., Gerla, M. "Queuing Network Models for Load Balancing in Distributed Systems", Journal of Parallel and Distributed Computing, Vol. 12, 1991.

[9] Walrand, J. "A Note on "Optimal Control of a Queuing System with two Heterogeneous Servers"", Systems and Control Letters, North Holland, 1984.

[10] Zhou, S. "A Trace Driven Simulation of Dynamic Load Balancing", IEEE Transactions in Software Engineering, Vol. 14, No. 9, May 1987.

[11] Leon-Garcia, A. "Probability and Random Processes for Electrical Engineering", Addison Wesley Publishing Company, 1989. 